\title{
E1 Nanocirco: un diseño interdisciplinario para la divulgación y enseñanza de la nanociencia y la nanotecnología
}

\author{
Bonny M. Ortiz-Andrade \\ Universidad de Puerto Rico, Recinto de Río Piedras: Instituto de Nanomateriales Funcionales, Programa de \\ Educación y Divulgación. San Juan.Puerto Rico.bonny.ortiz@upr.edu \\ ORCID: https:// orcid.org/0000-0001-5864-1482
}

Viviana Rivera-Rondón

Universidad de Puerto Rico, Recinto de Río Piedras: Instituto de Nanomateriales Funcionales, Programa de Educación y Divulgación.San Juan.Puerto Rico.viviana.riverarondon@upr.edu ORCID: https:/ / orcid.org/0000-0003-0910-6648

\section{Liz M. Díaz-Vázquez}

Universidad de Puerto Rico, Recinto de Río Piedras: Instituto de Nanomateriales Funcionales, Programa de Educación y Divulgación. San Juan. Puerto Rico.liz.diaz2@upr.edu

ORCID: bttps:/ / orcid.org/0000-0002-6285-0102

[Recibido: 8 Junio 2018. Revisado: 11 Septiembre 2018. Aceptado: 10 Febrero 2019]

Resumen: La nanociencia y la nanotecnología forman parte del desarrollo científico-tecnológico que contemplamos en la actualidad. Para educar en sus conceptos y diseminarlos en la sociedad se requiere de la creación de nuevas estrategias educativas. En este artículo describimos la investigación-acción realizada para la planificación, diseño y desarrollo de la obra el Nanocirco, en la que se integraron el circo y el teatro con la ciencia, para ofrecer una experiencia de comunicación científica no formal, motivar a las personas a la ciencia y enseñar cuatro conceptos de nanociencia y nanotecnología. Los hallazgos presentados son en su mayoría de carácter cualitativo, con algunos datos cuantitativos en términos de porcentajes. Estos fueron recopilados a partir de las reflexiones de los participantes del equipo de desarrollo Nanocirco, la evaluación de las funciones por parte de los espectadores y una preprueba y posprueba para evaluar el cambio en los estereotipos de los científicos. Los resultados sugieren el Nanocirco como un espectáculo interdisciplinario, motivador y generador de diversidad de instrumentos de enseñanza y comunicación, para los aprendizajes tanto del equipo desarrollador, como para los espectadores.

Palabras clave: Comunicación de la ciencia; Artes escénicas; Interdisciplinariedad; Estereotipos del científico; Diversidad de aprendizajes.

The Nanocircus: An interdisciplinary design for the dissemination and teaching of nanoscience and nanotechnology.

\begin{abstract}
Nanoscience and nanotechnology are part of the scientific-technological development that we contemplate today. To educate in their concepts and disseminate them in society, it requires the creation of new educational strategies. In this article, we describe the action research carried out for the planning, design, and development of the play The Nanocircus (El Nanocirco) in which the disciplines of circus and the theater were integrated with science to offer a non-formal scientific communication experience, to motivate the people to Science and teach four concepts of nanoscience and nanotechnology. The findings presented are mostly of a qualitative nature, with some quantitative data in terms of percentages. These findings were compiled from the reflections of the participants of El Nanocirco development team, the evaluation of the functions by the spectators and a pre-test and post-test to evaluate the change in the stereotypes of the scientists. The results suggest El Nanocirco as an interdisciplinary show, motivating and generating a diversity of teaching and communication instruments for the learning of both, the development team and the audience.
\end{abstract}

Keywords: Science communication; Scenic arts; Interdisciplinarity; Scientists stereotypes; Diversity of learnings. 
Para citar este artículo: Ortiz-Andrade B. M., Rivera-Rondón V., Díaz-Vázquez L. M. (2019) El Nanocirco: un diseño interdisciplinario para la divulgación y enseñanza de la nanociencia y la nanotecnología. Revista Eureka sobre Enseñanza y Divulgación de las Ciencias 16 (2), 2301. doi: 10.25267/Rev_Eureka_ensen_divulg_cienc.2019.v16.i2.2301

\section{Introducción}

En el siglo XXI la nanociencia y la nanotecnología (en adelante, NCT) han tomado protagonismo en el desarrollo científico-tecnológico. El prefijo "nano" de las palabras hace referencia al minúsculo tamaño en el que son fundamentadas y trabajadas. Generalmente entre 1 y 100 nanómetros (nm). El sistema internacional de medidas define un nanómetro como la millonésima parte de un milímetro, a esta escala es posible manipular átomos y moléculas (Brune et al. 2006). Por esto, a partir de la NCT se han podido concebir y modificar nuevos materiales que han acelerado el desarrollo y la creación de novedosos tratamientos para enfermedades como el cáncer, la producción de energía a partir de fuentes renovables, la remediación ambiental, la creación de dispositivos electrónicos con mayor capacidad de almacenamiento y velocidad, entre otros.

Por todos los beneficios y nuevos interrogantes que se derivaran de los avances en NCT, a nivel mundial se han creado diferentes centros para la investigación y educación en estas áreas. Por ejemplo, en Estados Unidos de América se creó en el 2000 la Iniciativa Nacional de la Nanotecnología (NSTC 2017) para ayudar a establecer colaboraciones entre científicos y acelerar su desarrollo. Este marco, ha generado varios retos y uno de los más grandes es la educación y divulgación de sus conceptos a la sociedad. Por otra parte, en el 2011 una amplia red de países iberoamericanos crearon la Red de Divulgación y Formación en Nanotecnología - nanoDYF, de manera similar, esta red tiene como objetivos el establecimiento de colaboraciones científicas y la divulgación de este contenido más allá de la comunidad científica (https://www.nanodyf.com).

El carácter innovador de la NCT requiere de la creación de nuevos currículos, actividades noveles de divulgación y estrategias de enseñanza-aprendizaje motivadoras y, para continuar su desarrollo, se requiere de personal especializado en este campo (Schwab 2016, Glenn y Florescu 2016, Yawson 2017). El no tener una fuerza laboral con los conocimientos y destrezas necesarias es la limitación más significativa para el desarrollo e implantación de la NCT (Roco 2011). Por tanto, una pieza clave en su futuro y desarrollo, es la educación científica y la formación de una nueva generación de personas capacitadas.

En este sentido, los objetivos de este estudio se alinearon a las necesidades educativas para el desarrollo y avance de la NCT. Específicamente, a la motivación de las personas por la ciencia y a la formación de una cultura científica mediante la enseñanza y comunicación de sus conceptos básicos. Este último, como parte de los saberes que los individuos deben poseer, para participar, opinar, tomar decisiones y dialogar de forma adecuada y sustentada sobre temas que pueden enmarcar situaciones académicas y de la vida diaria (Einsiedel y Goldenberg 2004, Laherto 2010).

En este artículo describiremos el proceso de planificación, diseño y desarrollo de la obra El Nanocirco, con la cual logramos diseminar y enseñar conceptos de la NCT. Este proyecto se diseñó para un contexto no formal, con el fin de motivar a la NCT y al aprendizaje tanto de espectadores, como de artistas y científicos que participaron. Para lograr este propósito y posibilitar un alcance diverso, en el Nanocirco se integró el circo y el teatro, con la ciencia. 


\section{Artes escénicas y comunicación de la Ciencia en contextos no formales}

Gran parte de las actividades de comunicación para la enseñanza de la Ciencia y la formación de cultura científica, son realizadas en contextos no formales de aprendizaje. Esto se debe a que su diseño posibilita una aplicación muy amplia; desde la noción de una audiencia pasiva hasta una experiencia totalmente participativa (Stocklmayer y Rennie 2017). Este tipo de actividades ofrece una excelente oportunidad de acceder a un público más diverso, facilita el desarrollo de otro tipo de actividades al no tener limitaciones curriculares y de horario escolar, hace posible la interacción con otras personas y facilita acceso a equipos que no están disponibles en las escuelas o que son difíciles de trasladar hasta el lugar (Fogg-Rogers, Weitkamp y Wilkinson 2015). Desde esta perspectiva, el diseño para un ambiente no formal goza de mayor flexibilidad, y las actividades y estrategias diseñadas pueden ser dirigidas a una diversidad de personas.

Como parte de la planificación de actividades no formales para la comunicación y enseñanza de la Ciencia, algunos trabajos exitosos se enfocan en el uso de las Artes escénicas. Entre estos, aquellos que integran las artes del circo y del teatro para llamar la atención y motivar a niños, jóvenes y adultos al aprendizaje. Un ejemplo es el estudio Kerby y colaboradores (2010), quienes describen un espectáculo que emplea las artes circenses en la enseñanza de la Química. Igualmente, el trabajo de Colley (2012) que sugiere la integración de las artes escénicas como herramienta innovadora para crear un ambiente idóneo para el aprendizaje. Otros autores como Chapela y Garritz (2013) proponen actividades similares, pero desde una perspectiva más inclinada a la divulgación de la ciencia. Los autores sugieren este tipo de estrategias no solo para formar nuevos científicos sino para combatir el analfabetismo científico. No obstante, estudios como los descritos son muy pocos y tienden a enfocarse exclusivamente en la comprensión del tamaño y la escala nanométrica. Tal es el caso de la exhibición interactiva Nano-Aventura del Museo Exploratorio de Ciencias de la Universidad de Campinas, en Brasil, la cual se desarrolla alrededor de dos videos narrados y cuatro juegos (Murriello y Knobel, 2008). Igualmente se describen en la web algunas muestras artísticas y proyectos de divulgación de la NCT, lamentablemente sin resultados publicados en revistas académicas que permitan realizar comparaciones efectivas. Dentro de estas iniciativas se pueden resaltar 1) la presentación "nanomandala", realizada en el 2004 por la artista Victoria Vesna y el científico James Gimsewsky, en donde nuevamente se alude, científicamente, a los conceptos de tamaño y escala, y 2) la obra NANOLAND, ideada por el Dr. Jordi Díaz de la Universidad de Barcelona, en la que con ayuda de payasos, marionetas y danzas, se presentan desde el 2007 diferentes conceptos de la NCT, como el tamaño, escala, confinamiento cuántico y superhidrofobicidad, entre otros.

\section{Metodología}

Empleamos principalmente una metodología cualitativa, con algunos datos cuantitativos en términos de porcentajes. Nosotros reflexionamos en todo el proceso de creación del espectáculo (planificación, diseño, implantación y evaluación), siempre con dirección al mejoramiento de nuestras prácticas y a la apreciación de la complejidad, diversidad y la riqueza del proceso implantado. Es decir, nuestra metodología tuvo como guía el diseño de investigación-acción, de tipo colaborativa (García-Carmona 2009). Proceso que propició la participación de espectadores, productores, actores, directores, entre otros, para el mejoramiento de prácticas educativas, en este caso, de la comunicación científica de NCT. Los datos fueron recopilados a partir los siguientes instrumentos:

a) Reflexiones grupales de los científicos/artistas: Realizadas durante la planificación y diseño del Nanocirco y al finalizar las funciones para evaluar el trabajo y aprendizaje 
en torno al crecimiento como profesionales.

b) Preprueba y posprueba: Para evaluar el cambio en la percepción de los espectadores sobre los científicos.

c). Evaluaciones del espectáculo por parte de los espectadores: Con una primera parte enfocada en la percepción de aprendizaje de los conceptos de NCT y en las herramientas ofrecidas por el Nanocirco que posibilitaron su aprendizaje y; una segunda parte enfocada la satisfacción de los espectadores y la motivación por la ciencia propiciada por el espectáculo

Los datos cualitativos fueron tratados mediante un análisis de narrativa para presentar al lector las diferentes experiencias del equipo Nanocirco y los espectadores, mientras que los datos cuantitativos fueron analizados en términos de porcentajes.

\section{Participantes}

Este proyecto logró impactar un total de 223 espectadores durante las funciones. Entre esta población se encontraban estudiantes de diferentes campamentos de verano y público general. La distribución de edades del público impactado se encontraba entre los 4 a 65 años. En la realización de la obra participaron un total de 65 colaboradores (Equipo Nanocirco), 35 fueron parte del elenco y producción [3 profesores, 1 director escénico, 6 niños, 6 estudiantes graduados (4 de química y 2 de educación en ciencias), 15 estudiantes subgraduados (3 humanidades, 1 arquitectura, 11 ciencias naturales), 4 artistas del circo] y 30 participaron como ujieres durante las funciones. Se realizaron 4 funciones en un teatro con capacidad para 60 personas, la asistencia media de espectadores por función fue de 56. La entrada al teatro fue libre y gratuita, siendo promocionada por radio, volantes, correo electrónico, redes sociales, periódicos digitales y página web institucional.

\section{Diseño del Nanocirco}

En un diseño interdisciplinario, plantear el propósito inicial es esencial (Boix-Mansilla 2016). En nuestro caso, apuntó al desarrollo de una obra teatral-circense para motivar a la NCT, mientras se enseñaba de manera divertida e interactiva sus conceptos básicos.

En la obra, el clásico debate entre la ciencia y la magia fue el hilo conductor que dio a conocer a los espectadores cuatro conceptos de la NCT utilizados en la National Science Fundación (NSF) (Stevens et al. 2007). Estos fueron:

a) Tamaño y escala: Es uno de los conceptos básicos en el entendimiento del mundo nanométrico. Por lo general, las personas definen el tamaño cualitativamente como grande o pequeño; sin embargo, en el campo científico es necesario definirlo a partir de escalas que permitan observar las diferencias entre los objetos macroscópicos, microscópicos y nanoscópicos.

b) Magnetismo: Es el fenómeno natural por el cual los objetos ejercen fuerzas de atracción o repulsión sobre otros materiales. Se empleó para explicar cómo las propiedades de la materia pueden cambiar cuando las partículas se encuentran en tamaño nanométrico.

c) Hidrofobicidad: Es la repulsión a las moléculas de agua. Este concepto en la nanociencia ha dado origen a diferentes nanotecnologías, que han cambiado la industria de los materiales como es el caso de la la industria textil y de la construcción.

d) Fluorescencia: Es el fenómeno por el cual algunas sustancias tienen la capacidad de absorber luz a una determinada longitud de onda, por lo general en el rango 
ultravioleta, y luego emiten luz en una longitud más larga. Este fenómeno se utiliza en la fabricación de instrumentos que son ampliamente utilizados en la caracterización de nanomateriales, así como en la fabricación de tintes fluorescentes para diferentes aplicaciones.

Para lograr el propósito planteado, por primera vez en nuestro Recinto se integró el conocimiento y las destrezas de dos facultades (Ciencias Naturales, Humanidades) y la comunidad artística representada por el Circo Nacional de Puerto Rico. Con este propósito se reclutaron estudiantes de ambas facultades a nivel graduado y subgraduado, y miembros de la comunidad artística. Nuestro trabajo documentó el proceso de aprendizaje e integración de diferentes disciplinas, la transferencia de conocimiento entre pares y las mejoras en las destrezas de comunicación.

El proyecto Nanocirco, se trabajó durante seis meses. Los tres primeros meses se dedicaron al desarrollo del concepto, libreto, escenografía; los subsiguientes tres meses se trabajó en el ensayo de la obra y el mejoramiento de elementos didácticos para una mejor enseñanza en escena. Durante todo el periodo se realizaron reuniones semanales en las que se propiciaban grupos de discusión y se proveía retroalimentación, entre los integrantes del equipo Nanocirco.

Por su carácter abstracto, los conceptos seleccionados fueron cuidadosamente estudiados y repensados para ser llevados a un público diverso, en el que niños, jóvenes y adultos se sintieran a gusto de aprenderlos. En este proceso, la integración de disciplinas jugó un papel importante, puesto que, para asumir los retos del diseño y planificación, las ideas fueron enriquecidas desde las distintas perspectivas de las disciplinas implicadas. De esta forma, las artes escénicas y la Ciencia brindaron conexiones que propiciaron la creatividad y la creación de estrategias e instrumentos únicos para motivar, comunicar y enseñar de manera adecuada los conceptos de la NCT. A continuación, presentaremos cada una de las estrategias e instrumentos diseñados.

\section{Libreto guía}

Este fue un instrumento inicial que sirvió de guía y creció durante el diseño y los ensayos del espectáculo. El libreto sigue una historia en la que el personaje principal (Tismo), descifra de manera divertida a través de sus conocimientos de nanociencia, los trucos del mago (Magne). Esto ocurre alrededor de cuatro actos, uno por concepto. En la historia, los personajes interactúan con el público, por lo que la llamada "cuarta pared" o división imaginaria de los actores y la audiencia fue eliminada. Se incluyen demostraciones, proyecciones y ejemplos cómicos de la vida cotidiana.

Durante el diseño del espectáculo, el diálogo constante y las reflexiones del equipo fueron claves para la evolución del libreto guía y el Nanocirco en general. A medida que avanzábamos asumimos varios retos, el desarrollo de artefactos acordes a los actos, la construcción de personajes, la adecuación del escenario de presentación, la elaboración de evaluaciones, la introducción de la preprueba y posprueba dentro del espectáculo, el desarrollo de materiales didácticos escritos y el desarrollo de una canción, entre otros.

\section{Interacción con el público}

En los actos los personajes interaccionaron con los espectadores, les realizaban preguntas, en ocasiones se les hizo partícipes de las discusiones entre la ciencia y la magia, les salpicaron de agua y nieve artificial, entre otros. Por ejemplo, durante el primer acto para representar/aprender el concepto de Tamaño y Escala, los presentadores invitaban a un espectador y mientras lo saludaban, uno de los presentadores hacía el que estornudaba y 
cubría la boca con su mano, con la cual saludaba luego al espectador. Entonces la Presentadora mirando con asombro la mano aparentemente sucia del participante exclama: ¡Que nadie se mueva! esta es una excelente oportunidad para mostrarles el mundo "nano". En seguida entra al escenario otro personaje con un instrumento grande y la presentadora sitúa la mano del espectador en el interior del instrumento. El presentador hace que mira dentro y exclama ¡Woow!... - En esta parte se apagan las luces para insertar otro tipo de estrategia didáctica: Los vídeos explicativos.

\section{Vídeos explicativos}

...Se apagan las luces e inicia una proyección en el escenario, se percibía como si se estuviera viendo aquella parte del estornudo que quedó en la mano. La proyección adentraba a los espectadores, en ella se visualizaba de mayor a menor escala desde el tamaño de un moco producto del estornudo, hasta el tamaño nanométrico de los puentes de hidrógeno del ADN presente en las bacterias que contenía el moco. De esta manera, los videos proyectados fueron parte de la función y se emplearon para ilustrar en el escenario los conceptos y objetos que no podían ser llevados al escenario. Por ejemplo, el mundo invisible del tamaño nanométrico y equipos como el microscopio de rastreo electrónico (SEM) y microscopía de fuerza atómica (AFM).

\section{Transiciones}

Fueron diseñados para mantener alerta al público, pero sin olvidar su concordancia con los conceptos puestos en escena. Tuvieron lugar entre los actos, de ahí el nombre de transiciones. Se presentaron acrobacias, malabares y bailes inspirados en los conceptos de tamaño y escala, hidrofobicidad y fluorescencia (figura 1).

\section{Canción nano}

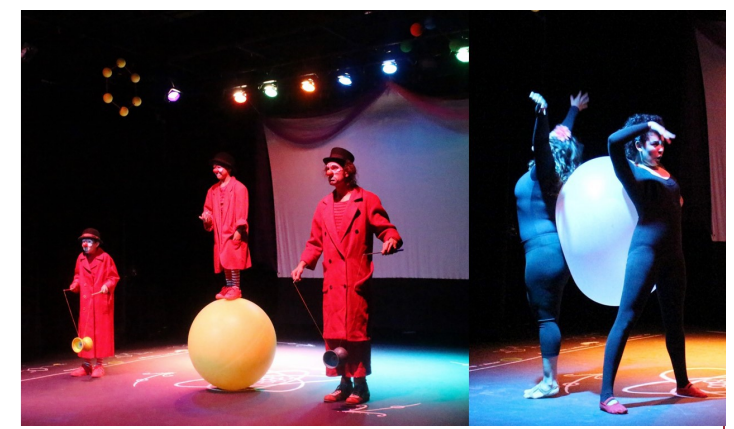

Figura 1. Transiciones para los conceptos tamaño yl escala e hidrofobicidad

tamaño y escala

Fue creada como refuerzo al concepto tamaño y escala y para animar al público, en especial a los niños asistentes. La canción se presenta por primera vez como transición en el acto II de la historia y se repite al final de la función acompañada por los movimientos del cuerpo de todos los personajes para el cierre del espectáculo. La canción complementa la historia y promueve el fortalecimiento de la memoria a través de sus ritmos y musicalidad. La letra de la canción "nano" lee como sigue

«Lo nano, enano no es.

Porque ni con este microscopio se ve. (dos veces)

En un metro hay 10 a la 9, y más pequeño el átomo es.

Átomo, átomo, todo está hecho de átomos (dos veces)».

\section{Los personajes}

Creamos cinco personajes dentro del marco referencial del libreto y con ayuda de un especialista en teatro y artes circenses. Estos fueron: dos presentadores, Tismo y Magne (representantes de la ciencia y la magia) y Zemog. Este último mostraba el estereotipo común del científico, el cual, para lograr evadirlo debíamos ponerlo en escena (tabla 1 y figura 2).

Aunque no formaban parte de la narrativa escrita en el libreto, otros participantes del equipo Nanocirco no menos importantes también hicieron parte del espectáculo. Malabaristas, bailarinas y payasos del circo; establecieron metáforas durante las transiciones y reafirmaron uniones entre las características artísticas de la ciencia y la ciencia que hay en el arte. Así 
mismo los NanoKids, fueron niños bailarines y portavoces de la canción nano durante la obra. Ellos alegraban las escenas, atraían la atención del público y con sus movimientos corporales daban sentido a la canción.

Tabla 1. Personajes y propósitos de acuerdo con sus roles en la obra teatral

\begin{tabular}{|l|l|}
\hline \multicolumn{1}{|c|}{ Personajes principales } & \multicolumn{1}{c|}{ Propósito } \\
\hline Presentadores 1 y 2 & Hacer primer contacto con la audiencia y definir conceptos pilares \\
\hline Tismo & $\begin{array}{l}\text { Representar la Ciencia y tratar de cambiar estereotipos y paradigmas a través de sus } \\
\text { acciones. Promover el rol de los científicos y el rol de la mujer en las Ciencias } \\
\text { Naturales }\end{array}$ \\
\hline Magne & $\begin{array}{l}\text { Representar el contraste entre la ciencia y la magia, estableciendo preguntas activas } \\
\text { y una voz dialógica desde la perspectiva de la "magia". }\end{array}$ \\
\hline Zemog "Científico Loco" & $\begin{array}{l}\text { Representar el estereotipo común del científico y parte de los elementos cómicos } \\
\text { en escena. }\end{array}$ \\
\hline
\end{tabular}

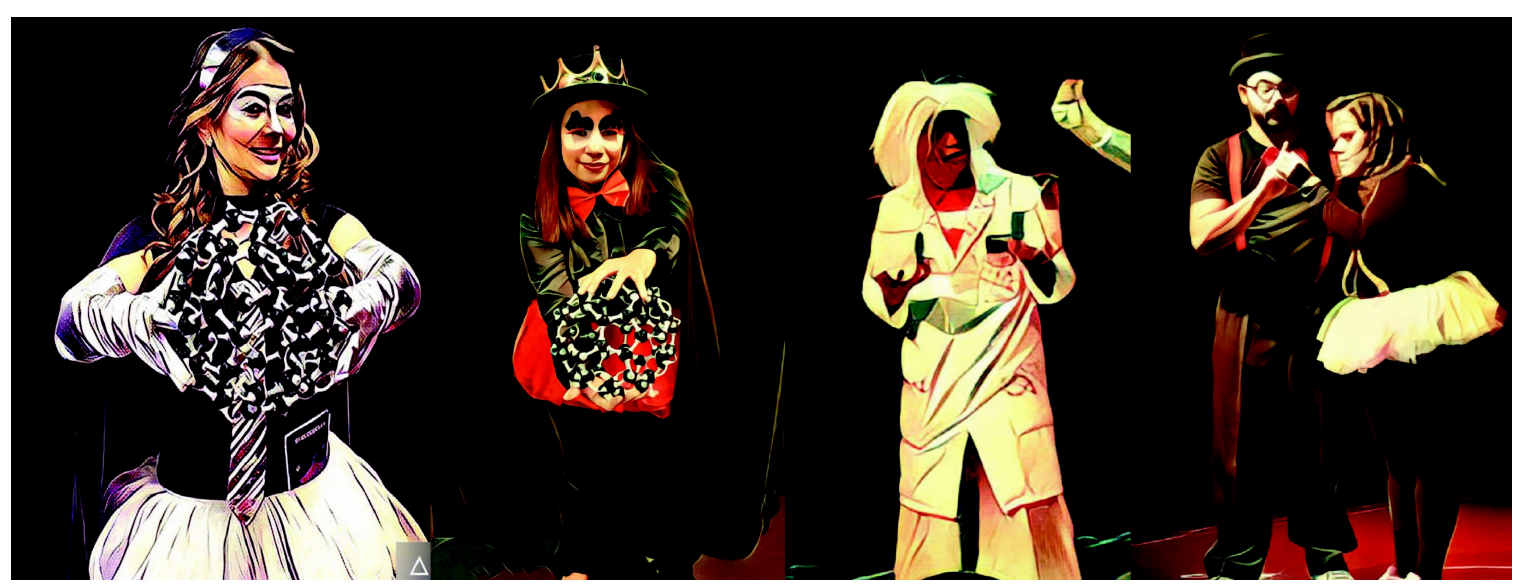

Figura 2. En orden de izquierda a derecha: Tismo, Magne, Zemog y presentadores

\section{Preprueba y posprueba}

Las diseñamos para evaluar el cambio en la percepción acerca de los científicos, pero también hizo parte del espectáculo. Antes del primer acto, cuando todos los espectadores se encontraban ubicados en el teatro, una de las líderes del proyecto tomaba la palabra para pedirles ayuda. Ella necesitaba elegir el científico que trabajaría con ella en su investigación de NCT. Luego les enseñaba un video con los personajes Tismo, Magne y Zemog. Los espectadores seleccionaban el personaje que creían, era el científico. De la misma manera, al finalizar la función con los personajes en el escenario, seleccionaban nuevamente su Científico. 


\section{Escenario interactivo}

El escenario fue preparado para que luego de finalizar el espectáculo el público pudiera interactuar en él. Se ilustraron diferentes moléculas biológicas y una escala con elementos propios de la NCT y elementos cotidianos (figura 3). De esta manera, el participante luego del espectáculo tenía la oportunidad de hacer un recorrido en búsqueda de información para completar una escala diseñada en uno de los materiales didácticos entregados (pasaporte interactivo) (anexo 1). Así, el escenario fue transformado en una pizarra interactiva.

\section{Demostraciones interactivas}

Similar al pasaporte interactivo, tuvo como propósito reforzar lo aprendido luego del espectáculo. Finalizada la función, los espectadores podían subir al escenario a interactuar con las demostraciones realizadas durante la función. Se situaron en mesas de exhibición, ferrofluidos, arenas hidrofóbicas, polímeros, elementos fluorescentes, entre otros. Cada mesa con una persona preparada para educar acerca de los conceptos y ofrecer respuesta a las preguntas de los niños y adultos de la audiencia.

\section{Hallazgos}

A continuación, presentaremos las reflexiones del Equipo Nanocirco, los resultados de la preprueba y posprueba en cuanto al cambio de percepción de los estereotipos de los científicos y la evaluación de las funciones por parte de los espectadores.

\section{Reflexiones de los científicos y artistas en el desarrollo de la obra}

El Nanocirco lo vimos crecer, nuestras ideas se veían materializadas gracias a la puesta en marcha de nuestra creatividad. A pesar de que teníamos las ideas bien pensadas (libreto, los personajes, los vídeos, la canción nano, el escenario interactivo) tuvimos que rediseñar escenas para que los materiales empleados como ferrofluidos, arenas hidrofóbicas, magnetos, materiales fluorescentes, lucieran como magia (demostraciones de Magne) y a la vez como ciencia (explicaciones de Tismo). ¿Cómo presentar el ferrofluido a los espectadores? ¿Cómo integrar las artes escénicas con los elementos científicos en escena? ¿Cómo hacer que el público comprendiera estos conceptos y a su vez se divirtiera? A reflexiones como estas durante el proceso de integración, logramos dar respuesta únicamente mediante las interacciones disciplinarias.

Unos aprendíamos de otros, los que tenían conocimiento del comportamiento de estos materiales de los que conocían la mejor manera de llevar elementos a escena y viceversa. No podíamos presentar el material como si estuviéramos en un espacio académico o un laboratorio y para esto era crucial que los que desarrollamos este proyecto creciéramos en las disciplinas que interaccionaban: La ciencia y las artes escénicas. Los artistas interactuaron con materiales que eran nuevos para ellos y se emocionaron la primera vez que los observaron (ferrofluidos, magnetos, arenas, hielo seco, entre otros). Hacían preguntas acerca de estos y a 
su vez aprendían de ciencia.

Así mismo, los que sabían de ciencia, debieron crecer en su presentación en escena. En este aspecto, las preguntas del director/artista fueron un aspecto clave para que notaran sus debilidades: ¿Por qué mueves tanto los brazos? ¿Esa es la mejor entrada que tienes? ¿Por qué agachas la cabeza? No conocían de este tipo de comunicación a pesar de que habían recibido cursos de comunicación de la ciencia. Fue un proceso de crecimiento en el que, según sus reflexiones, aprendieron a comunicar la ciencia de una manera diferente. El Nanocirco fue otra forma de aprender, otra forma de enseñar.

Las reflexiones de los que lideramos el proyecto también mostraron la importancia de esta integración para el éxito del Nanocirco. Planificar de manera integrada fue un reto, gracias a la participación de varios profesionales de distintas disciplinas, crecimos no solo en nuestra profesión, sino en cada una de aquellas de la que tuvimos que aprender para actuar, cantar y bailar los conceptos de la nanociencia y la nanotecnología.

Igualmente, los métodos y las acciones con los que comúnmente trabajaban artistas y las personas de Ciencia sugerían una transformación. Por ejemplo, los profesionales artistas al iniciar los ensayos marcaban su atención en los estiramientos, calentamientos y la relajación del cuerpo. Mientras que los profesionales de las ramas científicas centraban su atención en la adecuación y búsqueda de los materiales que se verían en la escena, tal y como acontece en el laboratorio. El director/artista manifestaba en sus reflexiones que lo más difícil de conseguir fue la disciplina durante los ensayos. Los científicos prestaban más atención a los materiales y a la utilería, que al calentamiento de su cuerpo que es la herramienta más importante en la escena.

Las reflexiones descritas revelan el crecimiento de las disciplinas durante el desarrollo del Nanocirco. Pero no fue un crecimiento aislado en el cual el artista profundizó únicamente en su baile o actuación, o en el que el científico profundizó en los temas de ciencia. Se trató de un crecimiento mutuo. Los científicos aprendieron otras formas de comunicar la ciencia gracias al arte y los artistas aprendieron conceptos científicos. Esto se observó en la ejecución de la obra y en el desarrollo de habilidades que los participantes manifestaron en sus reflexiones. Haber formado parte del Nanocirco influyó en ellos permitiéndoles ampliar y mejorar otras actividades que llevaron a cabo en cada uno de sus contextos profesionales.

Estas reflexiones muestran una integración clara entre las disciplinas para el crecimiento mutuo y sugieren además un proceso de transferencia. El emplear lo aprendido en otros contextos y situaciones.

\section{Preprueba y posprueba}

La percepción del científico por parte del público se presenta en la figura 4. Como se observa, antes de iniciar la función, la mayoría de las personas identificó correctamente a la científica en este rol. Sin embargo, el 51\% del público seleccionó incorrectamente en este rol a la Maga y a Zemog (figura 4A). Al finalizar la puesta en escena, la gran mayoría del público, $90 \%$, identificó correctamente a la científica.

Para comprender mejor estos resultados, en las figuras 4B y 4C se presenta la población segmentada en dos grupos. En cuanto a las edades (figura 4B), se observa que tanto adultos como niños presentan el mismo patrón de cambio de percepción. Ambos grupos migran hacia una identificación correcta de la científica mientras disminuyen los porcentajes atribuidos a los otros dos personajes. Aunque un alto número de adultos identificó correctamente al personaje en la prefunción, lo más notable fue que después de la función, los niños encontraron herramientas de juicio que les permitieron cambiar su opinión hacia una correcta 
identificación de la científica. En cuanto al género (figura 4C), se observó un patrón de percepción y cambio muy similar.

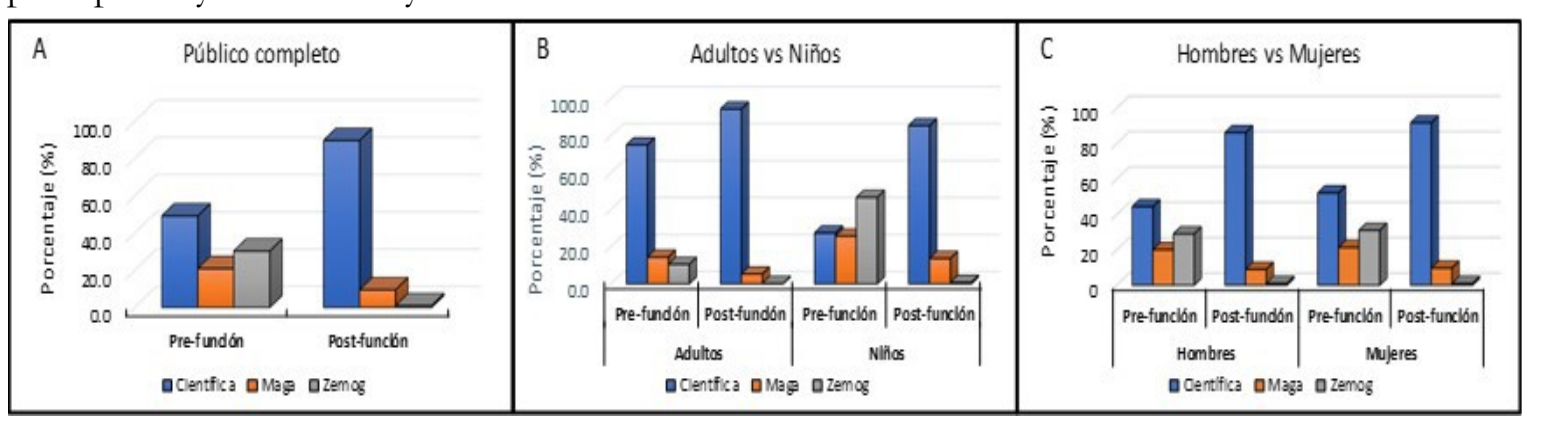

Figura 4. Cambio de percepción acerca del científico.

Antes de la función, los espectadores seleccionaron a su científico teniendo como criterio únicamente el video que mostraba su apariencia. Pero, en general, luego de la función la mayoría seleccionó a Tismo, es decir, la persona que en realidad representaba un científico. Lo que nos hace pensar que durante el espectáculo los espectadores asociaron al científico por las acciones y explicaciones fundamentadas acerca de los fenómenos presentados, más no por su apariencia, en este caso la apariencia clásica estereotipada del "loco de cabello blanco" que representó el personaje Zemog.

\section{Evaluaciones del espectáculo por los participantes}

Fueron completadas por 163 personas (niños y adultos). La parte cuantitativa de las evaluaciones expuso la satisfacción de los espectadores y la motivación por la ciencia propiciada por el espectáculo. La tabla 2 muestra las respuestas en términos de porcentajes.

Tabla 2. Evaluación cuantitativa.

\begin{tabular}{|l|c|c|c|c|}
\hline & $\begin{array}{c}\text { Excelente } \\
\text { (Super Wow) }\end{array}$ & Bueno & Interesante & Aburrido \\
\hline $\begin{array}{l}\text { ¿Cómo te pareció } \\
\text { el espectáculo? }\end{array}$ & $72,80 \%$ & $20,40 \%$ & $6,20 \%$ & $0,60 \%$ \\
\hline & \multicolumn{2}{|c|}{ Sí No } & No responde \\
\hline $\begin{array}{l}\text { ¿Recomendarías el Nanocirco a otras } \\
\text { personas? }\end{array}$ & $92 \%$ & $6,1 \%$ & $1,8 \%$ \\
\hline $\begin{array}{l}\text { E1 Nanocirco me } \\
\text { ayudó a... }\end{array}$ & Mucho & Bastante & Un poco & Para nada \\
\hline $\begin{array}{l}\text { Sentir que puedo } \\
\text { aprender ciencia }\end{array}$ & $72,8 \%$ & $4,3 \%$ & $22,8 \%$ & $0,0 \%$ \\
\hline $\begin{array}{l}\text { Aprender Ciencia de } \\
\text { Manera divertida }\end{array}$ & $88,3 \%$ & $3,1 \%$ & $8,6 \%$ & $0.0 \%$ \\
\hline $\begin{array}{l}\text { Querer aprender } \\
\text { más ciencia }\end{array}$ & $85,2 \%$ & $1,9 \%$ & $10,5 \%$ & $2.5 \%$ \\
\hline
\end{tabular}

Los resultados de la evaluación sugieren un espectáculo que satisfizo a los espectadores. El 72 $\%$ lo valoró como excelente (Super Wow) y el $92 \%$ lo recomendaría a otras personas. En cuanto a la motivación por la Ciencia se obtuvieron resultados similares. La mayoría de los evaluadores sintieron que eran capaces de aprender ciencia $(72 \%)$, aprendieron ciencia en el espectáculo $(88,3 \%)$ y quieren aprender más Ciencia $(85,20 \%)$. 


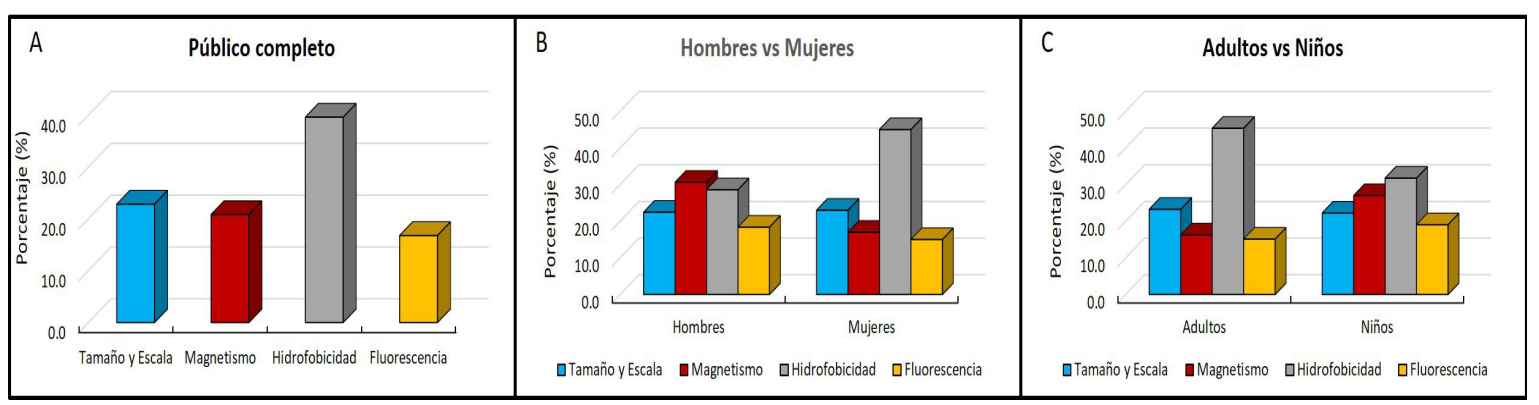

Figura 5. Percepción del concepto mejor comprendido por los espectadores.

Por otra parte, respecto a las perspectivas de aprendizajes, un gran número de espectadores seleccionó el concepto Hidrofobicidad como aquel que habían comprendido mejor (figura 5). Esto nos lleva a pensar que la comprensión de los conceptos puede estar relacionada con la variedad de elementos didácticos usados en cada acto. Puesto que los actos para Hidrofobicidad y Tamaño y escala, involucraron más variedad de estos elementos en el escenario (tabla 3).

En los resultados por grupos, tres sobresalen: 1) En el análisis por género, las mujeres manifestaron haber comprendido mejor el concepto de hidrofobicidad. Lo mismo fue cierto para los adultos en la clasificación por edades. 2) En los grupos por género y edad, los hombres y niños, respectivamente, presentaron una distribución más homogénea entre los conceptos, a su entender mejor comprendidos. 3) En cualquiera de las tres segmentaciones presentadas, la fluorescencia apareció como el concepto menos seleccionado.

Tabla 3. Elementos didácticos usados por cada acto para el aprendizaje de los concepto.

\begin{tabular}{|c|c|c|c|c|}
\hline & $\begin{array}{l}\text { Tamaño y escala } \\
\text { (Acto I) }\end{array}$ & $\begin{array}{l}\text { Magnetismo } \\
\text { (Acto II) }\end{array}$ & $\begin{array}{l}\text { Hidrofobicidad } \\
\text { (Acto III) }\end{array}$ & $\begin{array}{l}\text { Fluorescencia } \\
\text { (Acto IV) }\end{array}$ \\
\hline $\begin{array}{l}\text { Explicaciones en } \\
\text { escena }\end{array}$ & $\begin{array}{l}\text { Diálogo y preguntas } \\
\text { entre personajes }\end{array}$ & $\begin{array}{l}\text { Diálogo y preguntas } \\
\text { entre personajes }\end{array}$ & $\begin{array}{l}\text { Diálogo y preguntas entre } \\
\text { personajes }\end{array}$ & $\begin{array}{l}\text { Diálogo y preguntas } \\
\text { entre personajes }\end{array}$ \\
\hline $\begin{array}{l}\text { Etimología de } \\
\text { los conceptos }\end{array}$ & Canción nano & - & $\begin{array}{l}\text { Prefijo (Hidro-Hydro) y sufijos } \\
\text { (filia-Philia) y (Fobos-Phobos) }\end{array}$ & - \\
\hline $\begin{array}{l}\text { Elementos } \\
\text { cómicos }\end{array}$ & - & Zemog en acción & $\begin{array}{l}\text {...como mi perrito que es } \\
\text { hidrofóbico } \\
\text {...yo soy hidrofobica! }\end{array}$ & - \\
\hline $\begin{array}{l}\text { Proyección de } \\
\text { videos }\end{array}$ & 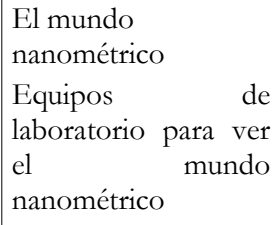 & - & $\begin{array}{l}\text { Gotas en superficies } \\
\text { hidrofóbicas }\end{array}$ & - \\
\hline $\begin{array}{l}\text { Interacción de } \\
\text { materiales con el } \\
\text { público }\end{array}$ & - & - & $\begin{array}{l}\text { Salpicar agua } \\
\text { Preguntas al público. } \\
\text { Magne hace caer nieve } \\
\text { artificial. }\end{array}$ & - \\
\hline $\begin{array}{l}\text { Demostraciones } \\
\text { en escena }\end{array}$ & $\begin{array}{l}\text { Espectador en } \\
\text { escena (jel achus!) }\end{array}$ & $\begin{array}{l}\text { Ferrofluidos, } \\
\text { magnetos, arenas. }\end{array}$ & $\begin{array}{l}\text { Arenas hidrofóbicas } \\
\text { Agua y hojas hidrofóbicas }\end{array}$ & Lluvia fluorescente \\
\hline $\begin{array}{l}\text { Transiciones de } \\
\text { circo }\end{array}$ & $\begin{array}{l}\text { Malabares - tamaños } \\
\text { de objetos }\end{array}$ & - & Baile gota de agua & $\begin{array}{l}\text { Malabares-Aro } \\
\text { fluorescente }\end{array}$ \\
\hline
\end{tabular}

De acuerdo con lo anterior, el concepto mejor comprendido por los espectadores fue también aquel que tuvo un mayor variedad de elementos didácticos. El concepto Hidrofobicidad contó con explicaciones en escena, explicación del concepto de acuerdo con su etimología, 
presentación de elementos cómicos y proyección de videos. Así mismo, se presentaron demostraciones del concepto, transiciones del circo y los espectadores interactuaron con algunos materiales. Es decir, se abordó desde una multiplicidad de estrategias que probablemente apuntaron a la diversidad de aprendizajes de la audiencia.

Lo anterior es además reflejado en la parte cualitativa de la evaluación. Cada espectador manifestó elementos particulares útiles para su aprendizaje, estos fueron identificados en las respuestas a las preguntas sobre el concepto favorito y el concepto mejor comprendido (véase la tabla 4).

Tabla 4. Comentarios acerca del concepto favorito y mejor comprendido.

\begin{tabular}{|c|c|c|}
\hline Espectador & $\begin{array}{l}\text { Concepto favorito } y \\
\text { mejor comprendido }\end{array}$ & Comentarios \\
\hline Estudiante, 11 años & $\begin{array}{l}\text { Fluorescencia y } \\
\text { tamaño y escala }\end{array}$ & $\begin{array}{l}\text { Porque ellos quieren que otras personas aprendan nano. Mi concepto } \\
\text { favorito fue fluorescencia porque es glow in the dark (brilla en la } \\
\text { oscuridad)y del que más aprendí fue tamaño y escala, porque ver desde una } \\
\text { gota, hasta el planeta tierra lo hizo más fácil. }\end{array}$ \\
\hline Estudiante, 9 años & $\begin{array}{l}\text { Hidrofobicidad y } \\
\text { Tamaño y escala }\end{array}$ & $\begin{array}{l}\text { Aprendí porque es muy divertido. El concepto que más me gustó fue el de } \\
\text { hidrofobicidad por que tiraron agua y aprendí mucho. El concepto que más } \\
\text { comprendí fue el de tamaño y escala. Cuando la gente estornuda, debería } \\
\text { taparse la boca. }\end{array}$ \\
\hline $\begin{array}{l}\text { Maestra de preescolar, } \\
37 \text { años }\end{array}$ & Hidrofobicidad & $\begin{array}{l}\text { A través de la diversión se aprenden conceptos de ciencia, en especial para } \\
\text { los niños y las niñas. Es una forma divertida y significativa de aprender. Por } \\
\text { la interacción con diferentes materiales, las imágenes y el video. }\end{array}$ \\
\hline $\begin{array}{l}\text { Ciudadano de la } \\
\text { comunidad, } 65 \text { años }\end{array}$ & Hidrofobicidad & $\begin{array}{l}\text { "Se aprende ciencia porque la usan para hacer reír y entretener - Concepto } \\
\text { favorito Hidrofobicidad y el que mejor comprendí. Ver como se repele el } \\
\text { agua en algunas substancias, hizo que se entendiera" }\end{array}$ \\
\hline Estudiante, 25 años. & $\begin{array}{l}\text { Fluorescencia e } \\
\text { hidrofobicidad }\end{array}$ & $\begin{array}{l}\text { Se explicaron los conceptos de forma sencilla y con ejemplos. Me gustan las } \\
\text { cosas brillantes. El concepto que mejor entendí fue hidrofobicidad, } \\
\text { explicaron la raíz de la palabra y también dieron buenos ejemplos. }\end{array}$ \\
\hline $\begin{array}{l}\text { Instituto de } \\
\text { investigación y } \\
\text { desarrollo de dotados, } \\
40 \text { años. }\end{array}$ & $\begin{array}{l}\text { Hidrofobicidad y } \\
\text { tamaño y escala. }\end{array}$ & $\begin{array}{l}\text { Se aprende mientras se divierte. Dio mucha gracia cuando tiraron el agua al } \\
\text { público. El concepto que mejor comprendí fue Tamaño y Escala por las } \\
\text { imágenes. ¡Excelente! los felicito, a mis niños les gustó mucho, deben hacer } \\
\text { más funciones para que más niños tengan la experiencia. }\end{array}$ \\
\hline $\begin{array}{l}\text { Educadora } \\
\text { Nivel elemental, } 43 \\
\text { años. }\end{array}$ & Todos & $\begin{array}{l}\text { Aprendemos a través de los sentidos. Se integró el teatro a la ciencia de } \\
\text { manera sencilla y divertida. Capturó mi atención }\end{array}$ \\
\hline $\begin{array}{l}\text { Estudiante, } \\
10 \text { años }\end{array}$ & $\begin{array}{l}\text { Hidrofobicidad y } \\
\text { Magnetismo }\end{array}$ & $\begin{array}{l}\text { Aprendí, porque repasé lo de los átomos. El concepto que más me gustó fue } \\
\text { el de hidrofobicidad, me pareció más cool. El que más aprendí fue el de } \\
\text { magnetismo, por las demostraciones. }\end{array}$ \\
\hline $\begin{array}{l}\text { Ciudadano de la } \\
\text { comunidad, } 43 \text { años }\end{array}$ & Hidrofobicidad & $\begin{array}{l}\text { Se aprende por la forma en que se presentan los conceptos. Fenómenos } \\
\text { relacionados a la nanoescala y materiales. Mi concepto favorito y el que } \\
\text { mejor comprendí fue hidrofobicidad, por los visuales, porque fue el más } \\
\text { interactivo, y con más experimentos. ¡Excelente labor! ¡Hace falta más } \\
\text { iniciativas como estas! }\end{array}$ \\
\hline $\begin{array}{l}\text { Educadora } \\
\text { Nivel secundario, } 30 \\
\text { años }\end{array}$ & Hidrofobicidad & $\begin{array}{l}\text { Se aprende ciencia por la dramatización, los videos, los ejemplos visuales. Mi } \\
\text { concepto favorito fue hidrofobicidad, es hermoso como el agua no llega a } \\
\text { mojar la hoja, también fue el concepto que mejor entendí por el video. } \\
\text { "Quiero que vayan a mi escuela" Se enseña de manera creativa y } \\
\text { emocionante temas sobre la ciencia. Cautivaron a la audiencia. ¡Bravo! }\end{array}$ \\
\hline Estudiante, 14 años & Hidrofobicidad & $\begin{array}{l}\text { Se aprende de ciencia por que muestran temas nuevos de forma interesante. } \\
\text { Mi concepto favorito, fue del que más aprendí: hidrofobicidad, por el } \\
\text { ejemplo de la arena y la hoja. }\end{array}$ \\
\hline $\begin{array}{l}\text { Representante } \\
\text { industria farmacéutica, } \\
45 \text { años }\end{array}$ & $\begin{array}{l}\text { Hidrofobicidad y } \\
\text { fluorescencia }\end{array}$ & $\begin{array}{l}\text { Lleva a los conceptos científicos de manera divertida y visualmente atractiva. } \\
\text { El hielo seco fue tremendo y las partículas fluorescentes muy bueno. }\end{array}$ \\
\hline
\end{tabular}


Los comentarios anteriores son una muestra de las diferentes formas en que los conceptos llegaron al público diverso, para el cual se habían diseñado diversas estrategias. La manera divertida, los visuales empleados, la interacción con diferentes materiales, la interacción con el público, los ejemplos en escena, las raíces de las palabras de los conceptos a explicar, la estimulación de los sentidos, las demostraciones, la canción nano, entre otras, fueron elementos por los cuales los conceptos lograron llegar al público y a la diversidad de aprendizajes.

Por otra parte, durante el espectáculo observamos que las reacciones que presentaba la audiencia en las diferentes escenas sugerían un tipo particular de evaluación, en ellas se podía evidenciar la comprensión de los conceptos, sobre todo en la ejecución de los elementos cómicos. Por ejemplo, en una de las escenas, luego de que el personaje Tismo explicara el concepto hidrofobicidad, Magne decía ingenuamente: ...como mi perrito que es hidrofóbico también!. Este elemento cómico únicamente causaría su efecto si la audiencia había entendido el concepto. Era un feedback inmediato para los que estaban actuando. Si los espectadores no se reían, el concepto no había quedado claro.

\section{Discusión}

El Nanocirco fue exitoso, logró la atención de los espectadores, fue una experiencia educativa innovadora que motivó a los espectadores al aprendizaje de la NCT. Estos hallazgos son similares a los presentados en otros proyectos que integraron la Ciencia y las Artes escénicas. Por ejemplo, en el llevado a cabo por Kerby y colaboradores (2010), en el que la integración de las artes escénicas llamó la atención de los espectadores, motivándolos al aprendizaje de la Química. De igual manera, hallazgos similares son descritos por Chapela y Garritz (2013), en su estudio afirman la importancia de este tipo de estrategias para motivar a la ciencia y de esta forma ganar cultura científica. Como en el Nanocirco, los hallazgos presentados por Colley (2012) y Blanco Martínez y González Sanmamed (2015), sugieren también la enseñanza mediante la integración de las artes escénicas como una herramienta innovadora que crea un ambiente idóneo para el aprendizaje. Nanoland es el proyecto de divulgación científica que más se asemeja al Nanocirco, tanto en el enfoque científico de la NCT y del arte escénico empleado para diseminar la información, como en la abundancia de actos y conceptos divulgados. Lamentablemente, a la fecha no encontramos registros en revistas académicas que nos permitan conocer su impacto en los participantes y espectadores.

En el diseño y ejecución del proyecto Nanocirco, las disciplinas ciencia y arte presentaron dos niveles de integración. Según Piaget (1975), existen tres niveles de integración de las disciplinas: Un primer nivel Multidisciplinario, en el que varias disciplinas aportan información para solucionar un problema; un segundo nivel Interdisciplinario, en el que la cooperación entre varias disciplinas lleva a interacciones reales, es decir, hacia una relación de intercambios que dan como resultado un enriquecimiento mutuo y, un tercer nivel Transdisciplinario donde ya no es posible distinguir con nitidez las fronteras entre disciplinas. Según esta clasificación, el Nanocirco inició en un nivel Interdisciplinario en el que hubo crecimiento mutuo a partir de las interacciones entre científicos y artistas. Sin embargo, el producto de la integración, es decir, el espectáculo final presentado, avanzó a un nivel Transdisciplinario. En su ejecución, el espectáculo lució como uno solo. Las disciplinas no parecían tener un límite claro, no sabíamos en donde dejaba de llamarse arte escénico para llamarse ciencia o tecnología. Si alguna de las disciplinas integradas no estuviera, el Nanocirco perdería su esencia.

La acción de planificación y diseño interdisciplinario del Nanocirco generó aprendizajes en los integrantes del equipo y luego, procesos de transferencia de lo aprendido a otros contextos y situaciones. De acuerdo con Alexander (2006) estos procesos de transferencia son un 
indicativo de la calidad del aprendizaje y, para Boix-Mansilla (2006) son además un resultado común de los procesos interdisciplinarios que indican un aprendizaje profundo. En palabras de Boix-Mansilla (2016 p 3): «El aprendizaje profundo implica la capacidad de "transferencia", es decir, la capacidad de utilizar información recién aprendida, en una situación nueva. Los principios de aprendizaje y los marcadores de calidad de este tipo proporcionan una sólida base genérica para la cognición interdisciplinaria». Los científicos y artistas del equipo, fueron capaces de emplear la información aprendida en situaciones nuevas de aprendizaje dentro de sus contextos.

En cuanto al aprendizaje de los conceptos, las respuestas de las evaluaciones sugieren una relación entre los conceptos mejor comprendidos con la diversidad de elementos didácticos del Nanocirco. De esta manera, la hidrofobicidad se presenta como el concepto mejor comprendido, mientras que fluorescencia aparece como el menos seleccionado. Estos hallazgos pueden tener su origen en las ocho inteligencias múltiples propuestas por Gardner (1998), las cuales se combinan e interaccionan dependiendo del contexto. Esto equivale a una diversidad inteligencias y por lo tanto, de aprendizajes en los espectadores, a los que difícilmente se llegaría con actividades homogéneas. Aunque este tema debe profundizarse en próximas investigaciones, Vivas (2015) afirma que no podemos pretender educar de manera homogénea, sino que debemos situar en la práctica métodos y diversas estrategias que posibiliten llegar a todas las personas con la misma eficacia. De la misma forma Ernst-Slavit (2001), sugiere a los educadores reconocer esta diversidad y ofrecer diversas experiencias de aprendizaje donde las inteligencias sean, estimuladas y desarrolladas.

Por otra parte, la explicación de la ciencia detrás de la magia por el personaje Tismo, contradecía el estereotipo común (Zemog) que rodó de escena en escena con incoherencias acerca de los conceptos. Esta historia logró su objetivo: contribuir a una visión adecuada de los científicos y animar al estudio de la Ciencia, especialmente, en la medida en que los niños, aquellos que inicialmente seleccionaron incorrectamente al personaje, tuvieron la oportunidad de cambiar la percepción inicial sobre la científica. Este hallazgo es particularmente útil puesto que según Pujalte, Bonan, Porro y Adúriz-Bravo (2014), el estereotipo común del científico influye en la baja motivación por la ciencia y en la selección de carreras científicas.

Adicionalmente, el desarrollo interdisciplinario del Nanocirco, ofreció oportunidades de evaluación diferentes. Por ejemplo, la preprueba y posprueba como parte del espectáculo y las reacciones del público (risas y carcajadas) como respuesta al entendimiento de los conceptos. Ante estas oportunidades de evaluación presentadas, Boix Mansilla (2006) y Boix-Mansilla y Duransing (2007) indican que es común que de los procesos interdisciplinarios surjan nuevas formas de evaluación.

\section{Conclusiones}

El Nanocirco logró diseminar y enseñar conceptos de la NCT. En su planificación, diseño y desarrollo interdisciplinario para un contexto no formal, se motivó a la ciencia y, tanto espectadores como artistas y científicos ganaron aprendizajes. En la interdisciplinariedad del Nanocirco las disciplinas interactuaron, se nutrieron y crecieron mutuamente. De esta forma, el espectáculo diseñado posibilitó múltiples elementos didácticos que sugieren el aprendizaje de conceptos en un público diverso. Se capturó la atención, se despertó el interés por la NCT y mientras los conceptos eran enseñados a través de los actos, los espectadores concibieron una visión más adecuada de los científicos.

El Nanocirco, no solo fueron funciones de enseñanza para un público diverso, sino que a partir de la experiencia, se convirtió en un proceso de aprendizaje para aquellos científicos y artistas que compartieron sus saberes. Los científicos desarrollaron habilidades de 
comunicación para enseñar de forma sencilla un contenido complejo y los artistas comprendieron conceptos de NCT, para comunicar lo aprendido mediante su arte.

El diseño no formal de integración de las artes escénicas y la ciencia, propició estrategias diferentes a las de la habitual enseñanza tradicional. Su diseño no contempla un maestro como principal actor del aprendizaje, son otros personajes que hacen reír, llaman la atención en sus presentaciones y desde el inicio (Preprueba) hasta el fin (demostraciones y pasaporte interactivo), el público hace parte del espectáculo. Esto creó de un ambiente único para divulgar y enseñar conceptos de NCT.

\section{Implicaciones}

El Nanocirco es un proyecto que puede ser inspiración para maestros, docentes y comunicadores científicos. El diseño descrito brinda ideas y nuevas perspectivas de enseñanza y comunicación de la NCT que pueden ser adaptadas a otros temas. Igualmente, este trabajo ofrece un marco para nuevas investigaciones; puesto que, aunque los espectadores evaluaron al Nanocirco y en el proceso ofrecieron sus perspectivas de aprendizaje. Posteriores investigaciones podrán abundar específicamente en la asimilación de los conceptos. Esta vez, evaluando en ellos (espectadores) la profundidad de lo aprendido.

Es necesario hacer notar que el Nanocirco, con todos sus participantes y elementos didácticos, no es una actividad de bajo costo. Sin embargo, en momentos de bajo presupuesto, la divulgación ha podido seguirse presentando de una manera fraccionada en la que se llevan a escuelas y comunidades demostraciones, videos, y escenas modificadas de los conceptos Magnetismo y Tamaño y Escala, que se acomodan a espacios, personal y utilería más sencillos.

\section{Agradecimientos}

A Puerto Rico NSF EPSCoR/RII Track-1 NSF Grant No. EPS-1002410. Agradecemos de manera especial al Circo Nacional de Puerto Rico y a su director Arturo Gaskins Rosado, Alba Taína Ortiz, Darell Y. Tirado y Diego N. Negrón. También agradecemos al Departamento de Drama de la Universidad de Puerto Rico, su director José Luis-Escobar, a los profesores Jorge Rodulfo, Israel Franco Müller y José A. Robledo González y a las estudiantes Melissa González Cotto y Yalitzaly Ramos. Agradecemos a todos los estudiantes colaboradores: Eddie Meléndez Merced, Arnulfo Rojas Pérez, Neikelyn Burgos, Emily Valentín Méndez, Kevin J. Torres Pérez, Jeremy Butler Rosario, Mireyssa Morales, Nadja Solis, Jaysha Infante, Orlando Salgado, Maryory Arzuaga, Christian Morales, Keyla Heredia, Cheysaliz Pérez, Adames y Edmary Santiago, Andrea Colón y Krystal López. A la Facultad de Educación, su decanato y al doctor William Padín; a al señor Jaime Figueroa del Teatro UPRRP; a los medios de comunicación: Radio Universidad y Diálogo UPR; y a todos los voluntarios que colaboraron de manera generosa.

\section{Referencias}

Alexander P. A. (2006) Psychology in learning and instruction. Englewood Cliffs, NJ. Prentice Hall.

Blanco Martínez A. B., González Sanmamed M. (2015) La perspectiva de alumnado de Educación Secundaria Obligatoria acerca de la utilización del teatro como recurso educativo. Magister 27 (2), 59-66.

Boix Mansilla V. (2006) Assessing expert interdisciplinary work at the frontier: an empirical exploration. Research Evaluation 15 (1), 17-29.

Boix Mansilla, V. \& Duraising, E. D. (2007) Targeted assessment of students' interdisciplinary work: An empirically grounded framework proposed. The Journal of Higher Education 78 (2), 215-237. 
Boix Mansilla, V. (2016) Interdisciplinary Learning: A cognitive-epistemological foundation. pp. 261-276 en Frodeman, R., Klein, J. T., Pacheco, R. C. D. S. (eds.) The Oxford handbook of interdisciplinarity, 2nd ed. Oxford. Oxford University Press.

Harald B., Ernst H., Grunwald A., Grünwald W., Hofmann H., Krug H., Janich P., et al. (2006) Nanotechnology: assessment and perspectives 27, Springer Science \& Business Media.

Chapela A., Garritz A. (2013) Ciencia en escena: Tres acercamientos a la Química. Educación quimica 24 (3), 262-267.

Colley B. (2012) Teaching social studies through the performing arts. The Educational Forum 76 (1), 4-12.

Einsiedel E. F., Goldenberg L. (2004) Dwarfing the social? Nanotechnology lessons from the biotechnology front. Bulletin of Science, Technology \& Society 24 (1), 28-33.

Erk S., Kiefer M., Grothe J., Wunderlich A. P., Spitzer M., Walter H. (2003) Emotional context modulates subsequent memory effect. Neuroimage 18 (2), 439-447.

Ernst-Slavit G. (2001) Educación para todos: La teoría de las inteligencias múltiples de Gardner. Revista de Psicología 19 (2), 319-332.

Fogg-Rogers L. A., Weitkamp, E. Wilkinson C. (2015) Royal Society education outreach training course evaluation. Project Report. Royal Society.

García Carmona A. (2009) La investigación-acción en la enseñanza de la Física: un escenario idóneo para la formación y desarrollo profesional del profesorado. Latin-American Journal of Physics Education 3 (2), 388-394.

Gardner H. (1998) Inteligencias Múltiples: la Teoría en la Práctica. Barcelona: Paidós.

Glenn J. C., Florescu E. (2016) Millennium Project Team (2016) Future Work/Technology 2050 Real-Time Delphi Study: Excerpt from the 2015-16 State of the Future Report. Journal of Socialomics 5 (3), 1000171.

Kerby H. W., Cantor J., Weiland M., Babiarz C., Kerb A. W. (2010) Fusion Science Theater Presents The Amazing Chemical Circus: A New Model of Outreach That Uses Theater To Engage Children in Learning. Journal of chemical education 87 (10), 1024-1030.

Laherto A., (2010) An analysis of the educational significance of nanoscience and nanotechnology in scientific and technological literacy. Science Education International 21(3), 160-175.

National Science and Technology Council (NSTC), Committee on Technology (COT) and Subcommittee on Nanoscale Science, Engineering, and Technology (NSET) (2017) The National Nanotechnology Initiative. Washington, D.C.

Piaget J. (1975) La epistemología de las relaciones interdisciplinarias. En Interdisciplinariedad. Apostel y otros, México, Biblioteca de la Escuela Superior, ANVIES.

Pujalte A. P., Bonan L., Porro S., Adúriz-Bravo A. (2014) Las imágenes inadecuadas de ciencia y de científico como foco de la naturaleza de la ciencia: estado del arte y cuestiones pendientes. Ciência \& Educação (Bauru) 20 (3), 535-548.

Roco M. C. (2011) The long view of nanotechnology development: The National Nanotechnology Initiative at 10 years. Journal of Nanoparticle Research 13, 427-445.

Schwab K. (2016) The fourth industrial revolution. Davos: World Economic Forum. 
Stocklmayer S. M., Rennie L. J. (2017) The Attributes of Informal Science Education: A Science Communication Perspective. pp. 527-544 en Preparing Informal Science Educators. New York. Springer.

Yawson R. M. (2017) Systematic Review to Identify Skill Needs for Agrifood Nanotechnology Workforce. Career and Technical Education Research 42(3), 149-181.

Vivas B. N. (2017) Las inteligencias múltiples como una estrategia didáctica para atender a la diversidad y aprovechar el potencial de todos los alumnos. Revista de Educación Inclusiva 8 (3) 121-136. 


\section{Anexo 1}

\section{Pasaporte Nanocirco}
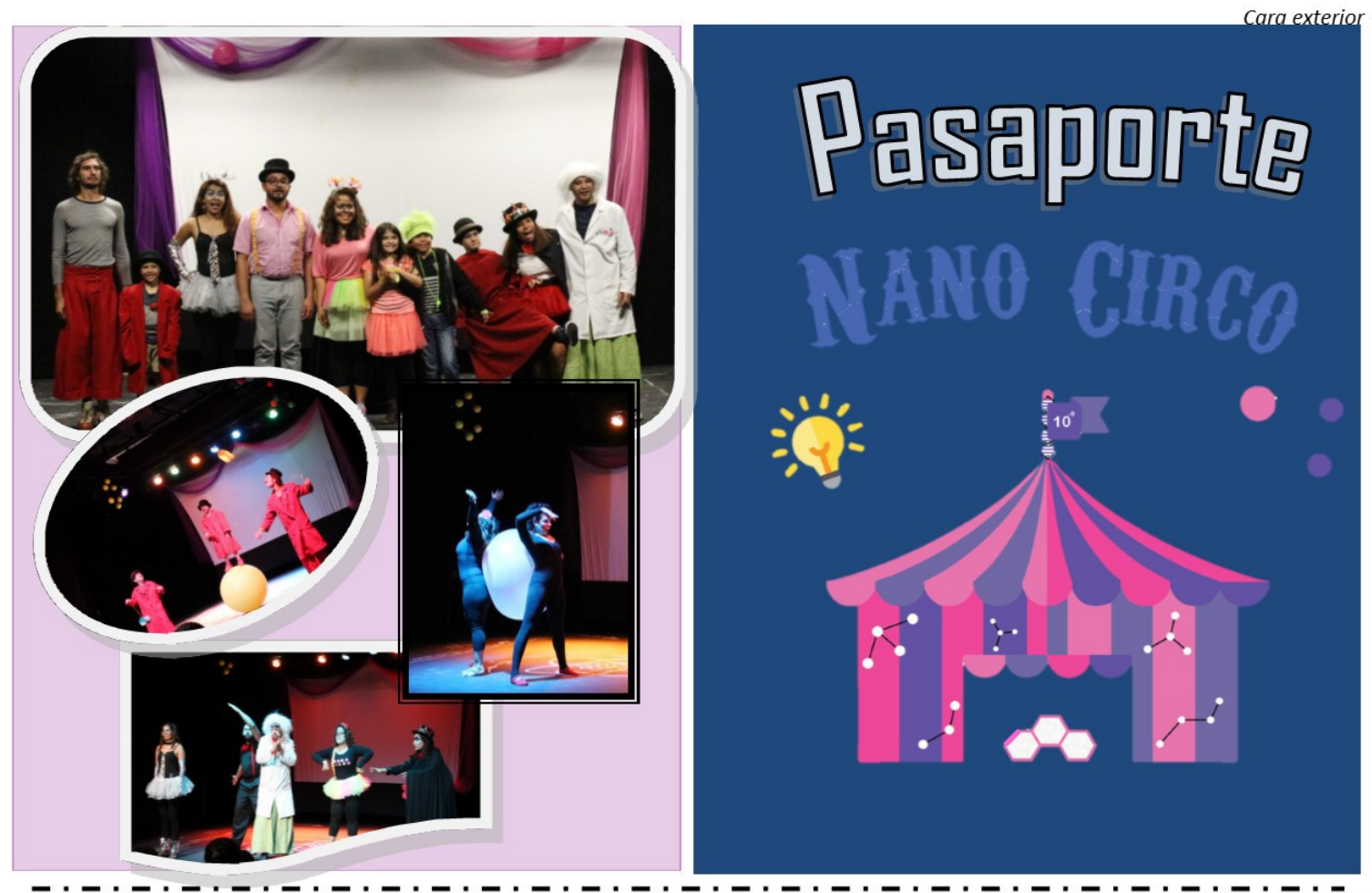

La nanotecnología es la manipulación de la materia a escala nanométrica. Nano es un prefijo griego que indica una medida $(10-9=0,000000001)$, no un objeto; de manera que la nanotecnología se caracteriza por ser un campo que une muchas disciplinas por la escala de la materia con la que trabaja.

¡El tamaño de la mayoría de las moléculas se encuentra en la región de 1 a $100 \mathrm{~nm}$. En la nanoescala del Nanocirco están representadas las moléculas de sustancias que provocan algunas de nuestras emociones, identifícalas en la siguiente tabla:

\begin{tabular}{|l|l|}
\hline Nombre de la molécula & Emoción \\
\hline & \\
\hline & \\
\hline & \\
\hline & \\
\hline
\end{tabular}

\section{¡Juguemos!}

Vamos a Explorar Juntos. En el escenario del Nanocirco hay una Nanoescala. Utilizala para completar Io que hace falta.

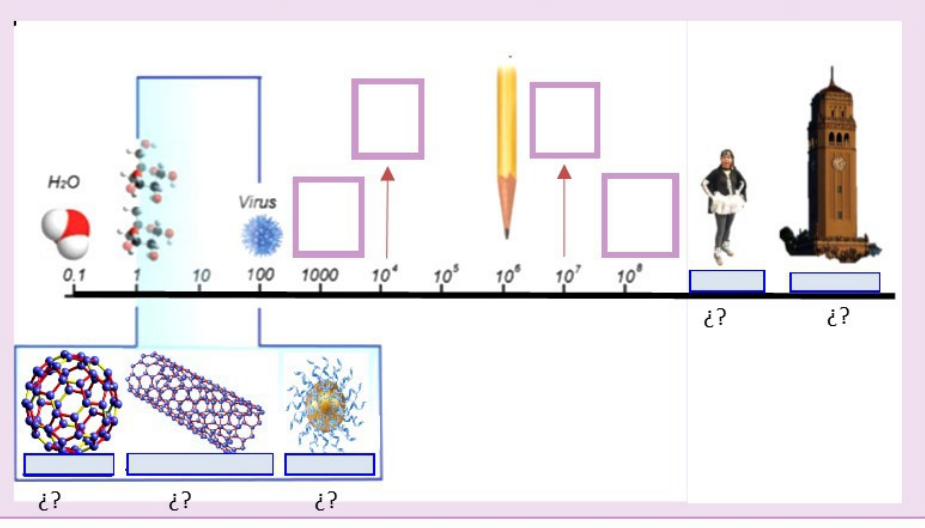




\section{Anexo 2}

Evaluación del Nanocirco por los espectadores

Edad Femenino Masculino

Escuela u organización

\section{NANO CIRCO}
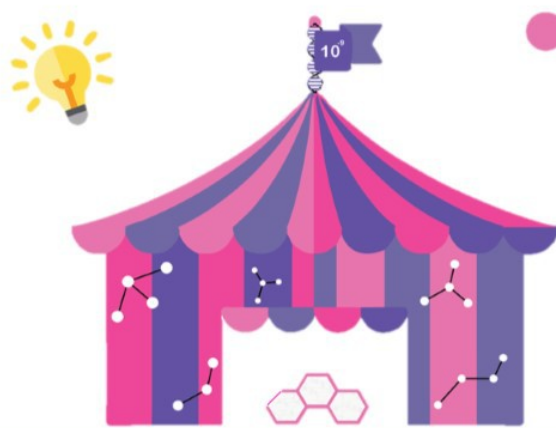

Ayudanos a mejorar nuestras próximas funciones con tu opinion.

Al respaldo encontrarás algunas preguntas sencillas acerca del espectáculo que observaste.

íGracias!

1. ¿Recomendarías el Nanocirco a otras personas?

Si $\square \quad \square$ No

2. Empleando tus propias palabras, responde las siguientes preguntas

¿Se aprende ciencia en el Nanocirco? Explica

3. Cuál fue tu concepto favorito.

$\begin{array}{ll}\text { Tamaño y escala } & \text { Magnetismo } \\ \text { Hidrofobicidad } & \text { Fluorescencia }\end{array}$

¿ेPor qué es tu concepto favorito?

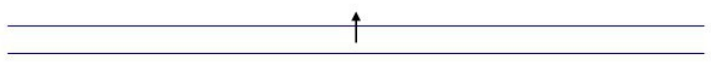

Ahora circula el concepto que entendiste mejor,

\section{Tamaño y escala}

Magnetismo

Hidrofobicidad

Fluorescencia
Déjanos saber cómo te sentiste en el Nano Circo

\begin{tabular}{|c|c|c|c|c|}
\hline & $\begin{array}{l}\text { Super } \\
\text { Wogy }\end{array}$ & Bueno & Interesante & Aburrido \\
\hline \multicolumn{5}{|l|}{$\begin{array}{l}\text { En general, el } \\
\text { espectáculo } \\
\text { te pareció }\end{array}$} \\
\hline $\begin{array}{l}\text { El } \\
\text { Nanocirco } \\
\text { me ayuda a }\end{array}$ & $\begin{array}{l}\text { Mucho } \\
\ddot{\theta}_{3}\end{array}$ & Bastante & Un poco & Para nada \\
\hline \multicolumn{5}{|l|}{$\begin{array}{l}\text { sentir que } \\
\text { puedo } \\
\text { aprender } \\
\text { ciencia }\end{array}$} \\
\hline \multicolumn{5}{|l|}{$\begin{array}{l}\text { aprender } \\
\text { ciencia de } \\
\text { manera } \\
\text { divertida }\end{array}$} \\
\hline $\begin{array}{l}\text { querer } \\
\text { aprender } \\
\text { más ciencia }\end{array}$ & & & & \\
\hline
\end{tabular}

¿Qué hizo que lo entendieras mejor? 